\title{
AUTOMATIC GAIN CONTROL IN THE BAT'S SONAR RECEIVER AND THE NEUROETHOLOGY OF ECHOLOCATION ${ }^{1}$
}

\author{
SHELLEY A. KICK AND JAMES A. SIMMONS ${ }^{2}$
}

Institute of Neuroscience, University of Oregon, Eugene, Oregon 97403

Received December 27, 1983; Revised May 25, 1984; Accepted May 30, 1984

\begin{abstract}
The sensitivity of the echolocating bat, Eptesicus fuscus, to sonar echoes at different time delays after sonar emissions was measured in a two-choice echo detection experiment. Since echo delay is perceptually equivalent to target range, the experiment effectively measured sensitivity to targets at different ranges. The bat's threshold for detecting sonar echoes at a short delay of only $1.0 \mathrm{msec}$ after emissions (corresponding to a range of $17 \mathrm{~cm}$ ) was $36 \mathrm{~dB}$ SPL (peak to peak), but the threshold decreased to $8 \mathrm{~dB}$ SPL at a longer delay of 6.4 msec (a range of $1.1 \mathrm{~m}$ ). Prior research has shown that, at even longer delays (corresponding to ranges of 3 to $5 \mathrm{~m}$ ), the bat's threshold is in the region of $0 \mathrm{~dB}$ SPL. Contractions of the bat's middle ear muscles synchronized with the production of echolocation sounds cause a transient loss in hearing sensitivity which appears to account for the observed echo detection threshold shifts.

The bat's echo detection thresholds increase by approximately $11 \mathrm{~dB}$ for each reduction in target range by a factor of 2 over the span from $17 \mathrm{~cm}$ to $1.1 \mathrm{~m}$. As range shortens, the amplitude of echoes from small targets also increases, by $12 \mathrm{~dB}$ for each 2 -fold reduction in range. Thus, when approaching a target, the bat compensates for changes in echo strength as target range shortens by changing its hearing threshold. Since this compensation appears to occur in the middle ear, the bat regulates echoes reaching the cochlea to a stable amplitude during its approach to a target such as a flying insect. In addition to this automatic gain control linked to target range, the bat aims its head to track a target's position during approach, thus stabilizing echo amplitude even if the target's direction changes. We hypothesize that the bat's directional emissions, directional hearing, middle ear muscle contractions, and head aim response collectively create a three-dimensional spatial tracking filter which the bat locks onto targets to stabilize echo amplitudes during interception of prey. We further hypothesize that this regulation, which cancels echo amplitude changes caused by the target's changing spatial position, leaves the bat free to observe echo amplitude changes caused by the target's own actions, such as insect wing beats. Elimination of spatially dependent echo amplitude changes removes the cause of potentially troublesome changes in neural response latency and keeps stimulation from echoes in the "tip" region of auditory nerve fiber tuning curves. The spatial tracking filter thus may stabilize the bat's acoustic images of a target's location and contribute to enhancement of the quality of these images.
\end{abstract}

Echolocating bats emit ultrasonic sounds to perceive objects in the environment. Echoes of these sounds return to the bat's ears, and the bat uses acoustic images derived from these echoes for spatial perception (Griffin, 1958; Novick, 1977; Simmons and Stein, 1980). Many species of echolocating bats are insectivorous and use their sonar to detect, locate, identify, and capture flying prey (Griffin, 1958; Simmons et al., 1979b; Simmons and Kick, 1983). To detect such small targets as flying insects at useful distances of several meters (Kick, 1982), bats often transmit very intense sonar signals. Peak-to-peak sound

\footnotetext{
${ }^{1}$ Written for T. T. Sandel, who showed us how to integrate ethological, psychophysical, and physiological thinking. This work was supported by National Science Foundation Grant BNS 80-13170 and by grants from the Whitehall Foundation and the Medical Research Foundation of Oregon. We thank D. R. Griffin, A. D. Grinnell, W. M. Masters, A. L. Megela, A. J. M. Moffat, H. -U. Schnitzler, and N. Suga for their critical comments.

${ }^{2}$ To whom correspondence should be addressed.
}

pressures of 100 to $110 \mathrm{~dB}$ SPL or more are frequently observed at short distances in front of the bat's mouth or nasal broadcasting organ (Griffin, 1958; Novick, 1977). These emissions are, in fact, so intense as to be near the limits for linear sound propagation in the atmosphere. Sounds of significantly greater strength would undergo harmonic distortion while traveling toward the target (Gallego-Juárez and Gaete-Garretón, 1983).

Bats possess mechanisms which protect their hearing from the full strength of their sonar transmissions. The sonar sounds themselves are projected primarily forward toward targets rather than back toward the ears (Pye, 1980; Schnitzler and Henson, 1980). Also, the bat's middle ear muscles contract at the time of vocalization to attenuate direct self-stimulation of the inner ear (Wever and Vernon, 1961; Henson, 1965; Suga and Jen, 1975). Still, the outgoing sonar signal is a fairly strong stimulus and might well affect the bat's ability to hear much weaker echoes returning from targets very shortly after the emission (Griffin, 1958). Targets at biologically relevant distances, which for the most part extend out to about $5 \mathrm{~m}$, return 
echoes at delays of $30 \mathrm{msec}$ or less. When echolocation was discovered, there was considerable concern about whether bats could hear the necessarily weak echoes returning from insectsized targets so soon after emissions, and several early theories of echolocation were proposed in part to account for this apparent difficulty (Nordmark, 1960; Pye, 1960; Kay, 1962). However, experiments in which bats used echolocation to detect obstacles to flight or flying insects clearly showed that bats have no great practical problem in hearing echoes (Griffin, 1958). The early neurophysiological experiments of Grinnell (1963) and of Suga (1964) demonstrated, too, that the bat's auditory system was indeed sensitive to weak sounds occurring immediately after stronger sounds, removing the need to worry about bats being deaf to echoes.

Contractions of the bat's middle ear muscles at the time of vocalization persist for 5 to $8 \mathrm{msec}$ after the emission (Henson, 1965; Suga and Jen, 1975), and their gradual relaxation could reduce the bat's sensitivity to echoes during this interval (Henson, 1967). Furthermore, neural events observed to be associated with vocalization suggest that bats have some central mechanism for inhibition of neural responses specifically at the time of generation of sonar signals which the bat itself emits (Suga and Schlegel, 1972). However, no attempt has been made to measure directly the bat's sensitivity to sonar echoes occurring shortly after vocalization, even though these physiological observations suggest that it would be worthwhile to do so. Accordingly, we report here measurements of thresholds for echo detection by the big brown bat, Eptesicus fuscus, at different echo delays. The results contribute significantly to organizing our understanding of relationships between the bat's echolocation behavior and characteristics of its sonar receiver.

\section{Materials and Methods}

Echolocating bats of the species Eptesicus fuscus (big brown bats; Chiroptera, Vespertilionidae) were used as subjects in these experiments. The bats were collected in the Willamette Valley of western Oregon and were maintained in the laboratory in a moist room at 22 to $25^{\circ} \mathrm{C}$ on a diet of mealworms (Tenebrio larvae).

The echo detection experiments, with echoes returned at controlled delays after the bat's emissions, were carried out using a two-choice simultaneous discrimination procedure adapted for studying echolocation (Simmons and Vernon, 1971). Electronically simulated echoes (replicas of the bat's own sonar signals) were delivered to the bat as stimuli using a target simulator system described elsewhere (Simmons, $1973,1979)$. Testing took place in a closed room (3.8 m long, $2.5 \mathrm{~m}$ wide, and $2.9 \mathrm{~m}$ high) that was soundproofed and made anechoic at ultrasonic frequencies by covering the walls, ceiling, and floor with convoluted polyurethane foam sheets. Ambient noise at ultrasonic frequencies was too low to measure with our equipment. Overall noise sound pressure must have been less than $10 \mathrm{~dB}$ SPL. Attenuation of reverberation exceeded $40 \mathrm{~dB}$ at frequencies above $20 \mathrm{kHz}$. The apparatus consisted of an elevated $\mathrm{Y}$-shaped platform on which the bat rested while emitting sonar sounds to scan for the simulated target. The bat's response was to crawl from its observing position on the central part of the $\mathrm{Y}$-shaped platform toward the simulated target and onto one of the two side arms of the platform, whichever was in the direction of the simulated target. The bat was rewarded with a piece of a mealworm offered in forceps for responding correctly.

At first, to prepare the bats for the experiments, each bat was trained in an earlier procedure (Kick, 1982) to detect a real target, a plastic cylinder $3.2 \mathrm{~cm}$ in diameter and $5.0 \mathrm{~cm}$ high, suspended vertically on a $0.15-\mathrm{mm}$-diameter nylon filament at a distance of about $20 \mathrm{~cm}$. The target was presented to the bat either on the left or on the right of the bat's observing position, and the bat was pretrained to approach this target by crawling toward it and thus onto the left or the right platform. When the bat would reliably detect the cylindrical target at better than 80 to $90 \%$ correct responses for several days in succession, with at least 20 detection trials per day, the target was moved away from the bat by a few centimeters and more trials were conducted. When the bat could reliably detect the cylindrical target as far away as $50 \mathrm{~cm}$, it was judged ready to reccive electronic echocs simulating the presence of a target, and the cylinder was removed.
The target simulator and the bat's behavior in echo detection trials are shown in Figure 1. Two Brüel \& Kjaer model 4135 condenser microphones were placed $10 \mathrm{~cm}$ in front of the bat's head in positions separated by an angle of $40^{\circ}$ when viewed from the bat's observing position at the center of the Y-shaped platform. The sonar signals emitted by the bat were recorded by the microphones, amplified and filtered with Rockland model 442 bandpass filters, and then delivered to electrostatic loudspeakers, each driven by a high-voltage transistor (Simmons et al., 1979a), for return to the bat as artificial echoes. The loudspeakers were placed $40^{\circ}$ apart at varying distances from the bat (see below). Only one of the signal-returning channels was activated on each trial so that a single target would be simulated either on the left or the right (Fig. 1). Trials were begun by placing the bat on the center of the Y-shaped platform. The bat would emit sonar sounds toward the left and right sides by scanning back and forth a few times with its head and then would respond by crawling onto the platform arm corresponding to the active simulator channel. Correct responses were rewarded with food, and the bat was left alone ("time-out") for about 30 sec when it made an incorrect choice. When the bat had consumed its reward, it was picked up and re-placed at the observing position for the next trial. (Bats readily adapt to this procedure by crawling or hopping onto the experimenter's hand for return to the center of the platform.)

The distance from the hat to each of the loudspeakers (Fig. 1) was adjusted to produce the desired delay in echo time-of-arrival after the bat's sonar emissions. The delay of echo stimuli consists of the time required for the sonar signals to travel $10 \mathrm{~cm}$ from the bat to the microphone $(0.29 \mathrm{msec}$ for a velocity of sound at $344 \mathrm{~m} / \mathrm{sec})$ and then the time required for the sound to return from the loudspeaker to the bat. The electronic delay between the microphone and the loudspeaker is neglected here because it is infinitesimally short. The loudspeakers were placed at distances of $25,54,75,100$, or $200 \mathrm{~cm}$ in different repetitions of the echo detection procedure. The corresponding times

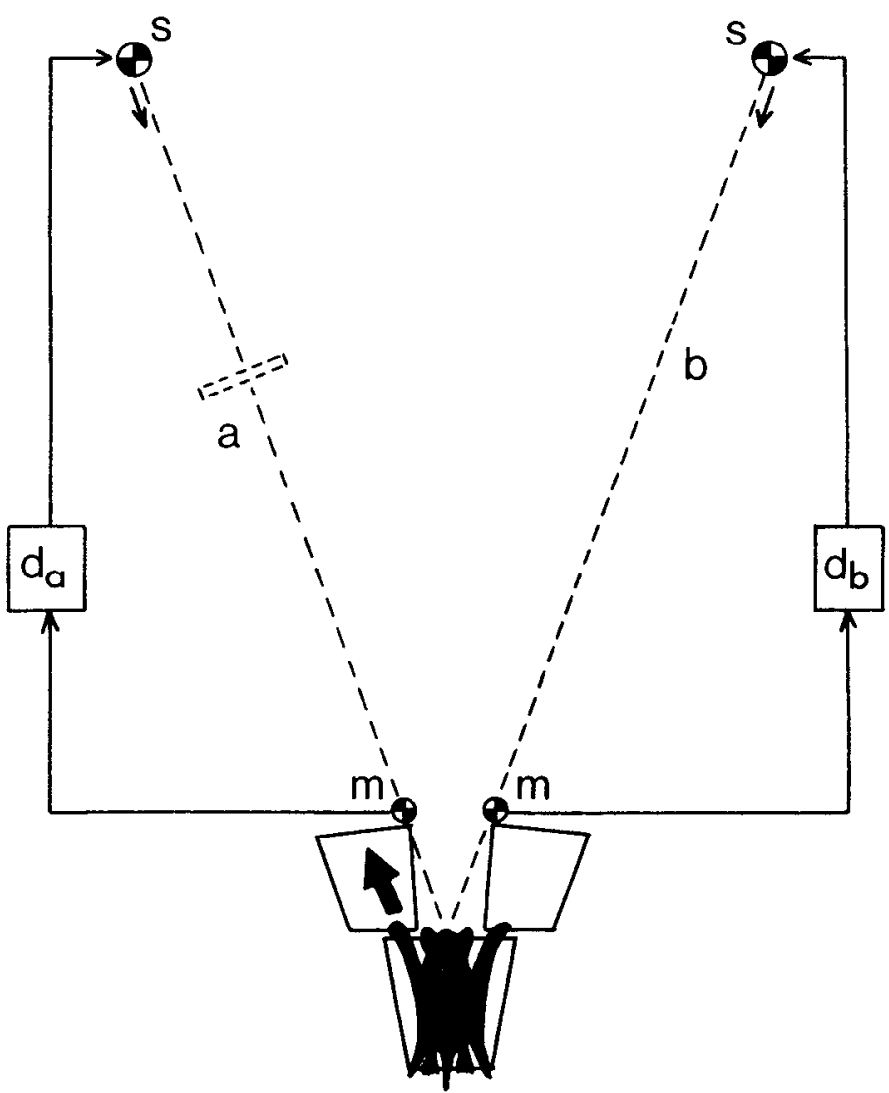

Figure 1. A diagram of the two-choice procedure and apparatus used for presenting the bat with electronic echoes in echo detection experiments. The bat's sonar signals are recorded with two microphones $(m)$ and conducted electronically to two loudspeakers $(s)$. Switches $\left(d_{a}\right.$ and $d_{b}$ ) permit operation of only one channel at a time. The bat is trained to respond by moving toward the side which presents the electronically simulated target $(a)$ and not to respond to the silent channel $(b)$. 
required for electronically delivered signals to travel from one of the loudspeakers back to the bat are $0.73,1.6,2.2,2.9$, or 5.8 msec. When the additional time required for the bat's sonar sounds to travel initially to the microphones is taken into account, the total time delay of electronic echoes presented to the bat was $1.0,1.9,2.5,3.2$, or $6.4 \mathrm{msec}$ These delays correspond to simulated target distances of $17,33,43,55$, and $110 \mathrm{~cm}$. The distances were chosen to span the range of echo delays that previous studies indicated would involve residual amounts of contraction of the bat's middle ear muscles (Henson, 1965; Suga and Jen, 1975).

Once they were trained to detect the cylindrical target as far away as $50 \mathrm{~cm}$ or so, the bats were transferred to electronic echoes as stimuli, with a delay of $2.5 \mathrm{msec}$ simulating a target at a distance of $43 \mathrm{~cm}$. The initial amplitude of the artificial echoes was set at 80 to $85 \mathrm{~dB}$ SPL (peak to peak), which was approximately the same as the amplitude of echoes produced by the pretraining target (the cylinder) at this distance. Using this electronically returned echo as the stimulus, the bats were then trained to choose reliably the side of the $\mathrm{Y}$-shaped platform which corresponded to the simulated target (the side corresponding to the acitve loudspeaker in Fig. 1). The presentation of the artificial echoes on the bat's right or left was varied from trial to trial according to a pseudorandom schedule used in previous two-choice experiments (Simmons and Vernon, 1971). Switches placed below the elevated platform controlled the location of the simulated target on the appropriate side of the apparatus by turning on one or the other of the two feedback channels.

When each bat learned to detect the electronic echoes delayed by $2.5 \mathrm{msec}$ at better than 85 to $90 \%$ correct responses for several consecutive days, the actual experiment commenced. The strength of the electronic echoes was reduced in 5- $\mathrm{dB}$ steps (using attenuators placed just prior to the power amplifiers and just after the power amplifiers to maximize electronic signal-to-noise ratios at the loudspeakers) to determine the bat's threshold for detecting the sounds. Beginning at a peak-to-peak sound pressure of $80 \mathrm{~dB}$ SPL, 20 to 50 experimental trials were conducted at each level in a progressively declining series of echo amplitudes until the bat's performance fell below the threshold criterion of $75 \%$ correct responses (halfway between chance performance at $50 \%$ and perfect performance at $100 \%$ ). Fifty trials were conducted at each amplitude level near the bat's threshold, and echo intensity decrements of $2 \mathrm{~dB}$ were sometimes used near threshold. After the threshold was determined at a simulated range of $43 \mathrm{~cm}$, the other distances were tested. These distances were presented to the bats in a mixed order to obtain a set of five threshold estimates for each bat. 'I'he raw data for each bat at each echo delay or simulated target range thus consisted of a percentage of correct responses on a block of 20 to 50 trials at each stimulus amplitude from $80 \mathrm{~dB}$ SPL (peak to peak) down, in 2- or 5- $\mathrm{dB}$ decrements, to an amplitude that was below the bat's echo detection threshold. These data were then reduced to the threshold estimates themselves for graphic display.

The sonar signals emitted by the bats during experimental trials were observed on a Non-Linear Systems model MS-15 portable oscilloscope mounted in easy view near the elevated platform used for the experiments. Echolocation signals used by each bat to detect stimuli at levels near threshold were recorded on a Racal Store-4 instrumentation tape recorder for later analysis with a real-time sound spectrograph specially constructed for analyzing the echolocation sound of bats. The two-channel acoustic recording and reproducing system that constituted the target simulator (Fig. 1) was calibrated using a Bruel \& Kjaer microphone placed at the bat's observing position on the Y-shaped platform. The frequency response of the simulator system from the point of reception of the bat's sonar emissions (the position of the microphones in Fig. 1) to the location of the bat's ears was flat to within $\pm 3 \mathrm{~dB}$ from 20 to $60 \mathrm{kHz}$. This frequency range contains the first-harmonic components of the sonar sounds of $E$. fuscus, which the bat uses for target detection (Simmons et al., 1978; Kick, 1982). The frequency response peaked from 30 to $50 \mathrm{kHz}$ to ensure that statements about the gain of the simulation system and the strength of echoes reaching the bat's ears came as close as possible to specifying conditions related to detection of echoes in natural conditions by the bat. From 20 to $100 \mathrm{kHz}$ the system's frequency response was flat to within \pm 8 $\mathrm{dB}$. Stimuli delivered to the bat's ears were characterized as having a particular peak-to-peak sound pressure at 30 to $40 \mathrm{kHz}$, which corresponds both to the frequency region emitted most strongly by the bat and to the region of strongest response of the simulator system. It should be remembered that the final stimulus magnitudes can only be known from the levels of the sonar signals emitted by the bats during the experiments. This was the reason for making tape recordings during selected trials near the bat's threshold.

\section{Results}

Four individual bats completed the echo detection threshold measurements at simulated target ranges from 17 to $110 \mathrm{~cm}$, except that one bat became sick and never completed the threshold measurement at a range of $55 \mathrm{~cm}$. Figure 2 shows the performance of one bat on a descending series of stimulus amplitudes at each of the five different echo delay times which were simulated electronically. This bat's data are representative of the data from the other bats in the experiments. The curves in Figure 2 illustrate how the bat's sensitivity to echoes depends upon ccho delay. For example, at a delay of $6.4 \mathrm{msec}$ the bat's threshold for echo detection at a criterion of $75 \%$ correct responses is approximately $6 \mathrm{~dB}$ SPL peak to peak. At a delay of $1.9 \mathrm{msec}$ the threshold is $31 \mathrm{~dB}$ SPL, indicating a loss in sensitivity. Curves such as those shown in Figure 2 are used to estimate thresholds at each delay value for each bat, and it is these thresholds at different delays which constitute the primary results of the experiments. As Figure 2 reveals, there is in fact considerable change in the bat's threshold for hearing echoes depending upon echo delay.

The threshold estimates depend upon the strength of the sonar signals produced by the bats. The sonar signals emitted by the bats during near-threshold detection trials were similar to the echolocation sounds previously observed to be used by E. fuscus in target detection experiments (Simmons et al., 1978; Kick, 1982). The signals were frequency modulated (FM) and contained two equal-strength harmonic components: a fundamental or first harmonic sweeping from 60 to $65 \mathrm{kHz}$ down to $30 \mathrm{kHz}$ and a second harmonic sweeping from $120 \mathrm{kHz}$ down to $60 \mathrm{kHz}$. The peak-to-peak sound pressures ranged from 105 to $110 \mathrm{~dB}$ SPL, with $109 \mathrm{~dB}$ SPL being the most typical amplitude. Accordingly, an amplitude of $109 \mathrm{~dB}$ SPL at $40 \mathrm{kHz}$ was used as the basis for acoustic calibration of the amplitude of stimuli actually delivered to the bats in these experiments.

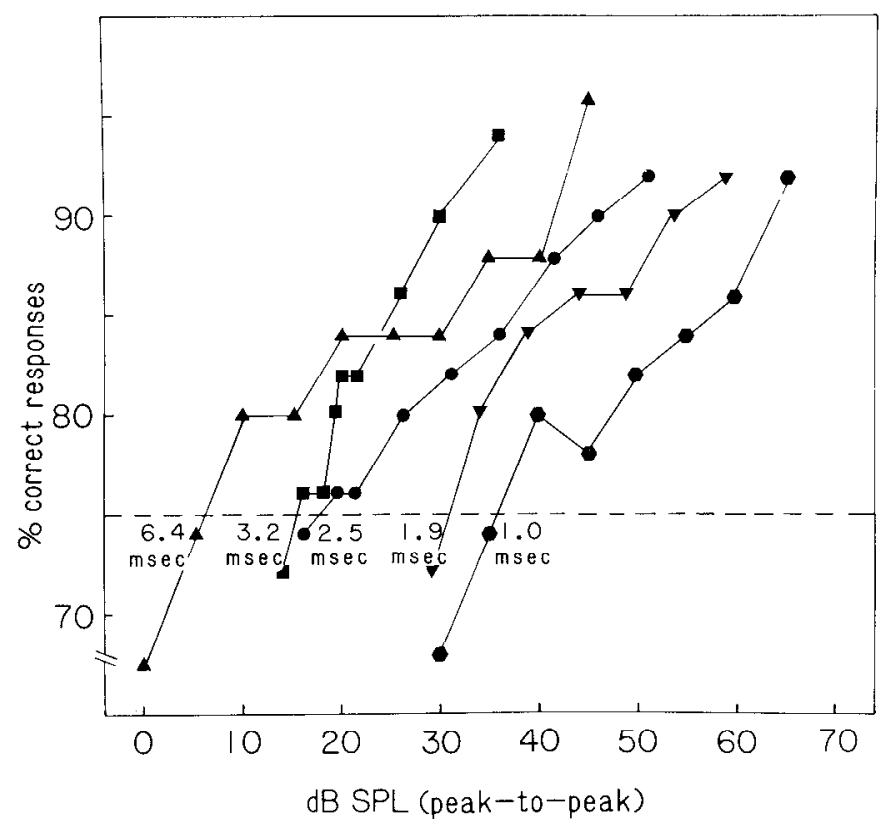

Figure 2. A graph showing the results of the echo detection experiment for an individual bat at five echo delay values from 1.0 to 6.4 msec. The bat's echo detection thresholds are determined from the points where these curves cross the level of $75 \%$ correct responses (horizontal dashed line). The curves reveal a general improvement in the bat's sensitivity to echoes as the delay of echoes (or simulated target range) increases. 
Variations in the amplitude of sonar emissions of any of the bats during detection trials amounted to about $5 \mathrm{~dB}$, which is similar to the range of variation seen in threshold estimates among all four bats at any particular echo delay.

Figure 3 shows the echo detection threshold estimates for each bat ( solid data points) at different echo delays or simulated target ranges (horizontal axes). ('The graph in Fig. 3 contains a number of additional pieces of information to be discussed below. For the present, ignore the line marked point target, for example.) The solid circles, triangles, diamonds, and squares in Figure 3 indicate for different bats the echo detection thresholds obtained in the experiments conducted here. These thresholds (vertical axis) are expressed in terms of peak-to-peak echo sound pressure in decibels sound pressure level at $40 \mathrm{kHz}$, for a 109-dB SPL sonar emission. The variability inherent in each threshold estimate is determined partly by the number of trials going into the threshold estimate, and this consists of the 50 trials at the stimulus attenuation setting yielding a performance just above $75 \%$ correct responses plus the 50 trials at the attenuation setting yielding a performance just below $75 \%$ correct responses. Stimulus attenuation was adjusted in 2- or 5 -dB steps, which produces a larger inherent variability in threshold estimates than does the 50-trial size of the data blocks (Grant, 1946). Thus, each threshold estimate probably is accurate to within about $5 \mathrm{~dB}$.

The echo detection thresholds obtained at shorter delays are substantially higher than thresholds obtained at longer delays. The mean echo detection threshold at a simulated range of 17 $\mathrm{cm}$ (referring to the lower horizontal axis of Fig. 3) is about 36 $\mathrm{dB}$ SPL (peak to peak), whereas the bat's threshold falls to about $8 \mathrm{~dB}$ SPL when the range increases to $110 \mathrm{~cm}$. The

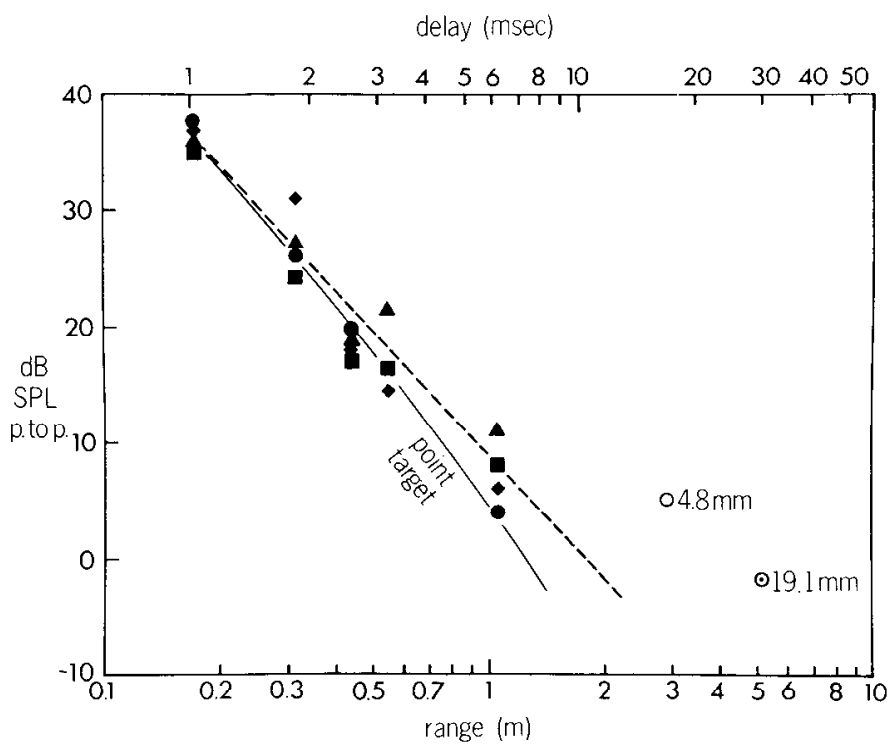

Figure 3. A graph showing the results of the echo detection experiments. The lower horizontal axis shows simulated target range in meters, the upper horizontal axis shows echo delay in milliseconds (assuming a velocity of sound in air of $344 \mathrm{~m} / \mathrm{sec}$ ), and the vertical axis shows peak-to-peak echo sound pressure in decibels sound pressure level at the bat's threshold. The solid data points show echo detection thresholds from experiments conducted using the procedure illustrated in Figure 1. The open data points show echo detection thresholds estimated from target detection experiments with $4.8-$ and $19.1-\mathrm{mm}$ spheres (Kick, 1982). The sloping dashed line is the regression line for the echo threshold data (solid data points). The sloping, slightly curved, solid line marked point target shows the amplitude of echoes from a small target at different distances from the bat (Lawrence and Simmons, 1982a). Only its slope is significant here; similar curves can be placed at different vertical positions to represent echoes from differentsized targets. decrease in echo detection thresholds over the span of echo delays tested in these experiments -1.0 to $6.4 \mathrm{msec}$ (referring to the upper horizontal axis of Fig. 3)-amounts to $28 \mathrm{~dB}$. Intermediate echo delays produce intermediate decreases in echo detection thresholds. The graph in Figure 3 hints at an approximately linear relationship between the bat's echo detection threshold expressed in decibels (that is, on a logarithmic scale) and the logarithm of target range. The regression line (dashed line in Fig. 3) calculated for the data points in the graph is

$$
y_{\mathrm{dB}}=-37 \log \text { (delay) }+36
$$

This regression line has a slope of $-11 \mathrm{~dB}$ for each increase in echo delay by a factor of 2 , between delays of 1.0 and 6.4 msec. The correlation coefficient for this relationship is -0.96 , which is strongly significant. The threshold for echo detection in $E$. fuscus is inversely dependent upon the delay of echoes after emissions, or upon target range.

\section{Discussion}

The results of the echo detection experiments shown in Figure 3 indicate that the sensitivity of $E$. fuscus to sonar echoes is not constant but does in fact improve as echoes occur at times increasingly remote from the sonar emission. Over a span of delays from 1.0 to $6.4 \mathrm{msec}$ the bat's sensitivity improves by almost $30 \mathrm{~dB}$. These delays correspond to target ranges from 17 to $110 \mathrm{~cm}$, which is significant, as will be seen below, since these distances encompass the most critical stage of the pursuit of flying insect prey by $E$. fuscus.

It is helpful to compare the bat's echo detection thresholds in the experiments reported here with threshold estimates obtained in previous target detection experiments (Kick, 1982). The echoes which $E$. fuscus receives from spherical targets at the maximum range of detection are about $5 \mathrm{~dB}$ SPL (peak to peak) from $4.8-\mathrm{mm}$ spheres at a range of $2.9 \mathrm{~m}$, and $-2 \mathrm{~dB} \mathrm{SPL}$ from $19.1-\mathrm{mm}$ spheres at a range of $5.1 \mathrm{~m}$. These estimated threshold values are shown in Figure 3 as open data points (circles) located in the lower right corner of the graph. Taken together, the echo detection thresholds measured here (Fig. 3, solid data points) and the echo magnitudes estimated from target detection experiments (Fig. 3, open data points) reveal a pattern of change in the bat's sensitivity to sonar echoes which depends upon the temporal proximity of echoes to the sonar emissions which produced them. The bat's threshold remains low if echoes return at delays greater than roughly 6 to $10 \mathrm{msec}$, or if the targets which return these echoes are at distances greater than about 1 to $1.7 \mathrm{~m}$. The bat's threshold for detecting echoes at these relatively long delays is in the region of $0 \mathrm{~dB}$ SPL (peak to peak). If echo delay is shorter than 6 to $10 \mathrm{msec}$, or if target range is shorter than 1 to $1.7 \mathrm{~m}$, the bat's threshold for detecting echoes rises by about $11 \mathrm{~dB}$ for each reduction in delay (or range) by half. At a delay of $1.0 \mathrm{mscc}$, or a range of $17 \mathrm{~cm}$, the bat's threshold may be as much as $36 \mathrm{~dB}$ higher than it is when targets are at relatively long ranges. Some event associated with the production of a sonar sound appears temporarily to reduce the sensitivity of hearing in $E$. fuscus, and the effect of this loss in sensitivity persists for a span of time that is biologically relevant to the bat. We examine below the consequences of this transient loss in hearing sensitivity for the process of echolocation.

Not only did the bat receive the electronically produced echoes in this experiment, but it also received echoes from various objects present in the experimental room. In particular, the faces of the loudspeakers which broadcast echoes back to the bat also reflected the bat's sonar emissions to return real echoes (see Fig. 1). Since these real echoes from the loudspeakers returned to the bat shortly after the artificial echoes which served as stimuli, they conceivably could have affected the bat's 
sensitivity to the electronic echoes. A control experiment was conducted to determine the size of the zone of target range within which echoes from one target can interfere with echoes from another target. This experiment developed a life of its own and is described elsewhere (J. A. Simmons, S. A. Kick, A. J. M. Moffat, and W. M. Masters, manuscript in preparation). The zone of clutter interference along the dimension of range is about $15 \mathrm{~cm}$ wide and is such that the real echoes from the loudspeaker may have affected the bat's sensitivity to artificial echoes in the condition of shortest delay $(1.0 \mathrm{msec})$ or shortest simulated range $(17 \mathrm{~cm})$, but not at other delays or ranges. The magnitude of the interference effect is small since the real echoes from the loudspeakers have an amplitude of $43 \mathrm{~dB}$ SPL (peak to peak) when at a distance of $25 \mathrm{~cm}$. This is only about $13 \mathrm{~dB}$ above the bat's threshold at a range of $25 \mathrm{~cm}$ and only a few decibels more than the echoes which the bat detects at its $36-\mathrm{dB}$ SPL threshold at a range of $17 \mathrm{~cm}$. Furthermore, when a bat has approached to within a distance of $17 \mathrm{~cm}$ from a target under natural conditions, it has reached the terminal stage of the interception process. During this stage, the bat's sonar emissions decline in amplitude by about $6 \mathrm{~dB}$ (Griffin, 1958; Webster, 1967), thus raising the bat's threshold for detecting sonar targets because the strength of the emission itself helps to determine the strength of echoes. (The repetition rate of emissions during the terminal stage also increases to more than 100 sounds/sec, altering the dynamic properties of the mechanism that appears to cause the loss in sensitivity to echoes shown in Fig. 3; see below.) The bats in our experiments, however, did not decrease the amplitude of their emissions when detecting echoes at short delays in the experiments reported here. Thus, the echo detection threshold shown in Figure 3 at a distance of $17 \mathrm{~cm}$ is about $6 \mathrm{~dB}$ too low compared to natural conditions because the bat would have used weaker sounds under natural conditions when this close to a target. It may be a few decibels too high because weak interfering effects could have occurred due to the echoes from the faces of the loudspeakers. At other, longer ranges, neither of these complications occur.

Automatic gain control for target range. The strength of echoes returning to an echolocating bat from a target depends upon the target's range. The nearer the target, the stronger the echoes. As range increases, the strength of echoes declines by an amount determined by the nature of the acoustic wavefront of the bat's sonar emission, by the nature of the wavefront scattered by the target, and by atmospheric absorption of sound (Griffin, 1958, 1971; Pye, 1980; Lawrence and Simmons, 1982a). Spreading losses for the emission and the echo from a small target reduce the strength of echoes by a total of $12 \mathrm{~dB}$ for every doubling of target range. The effects of atmospheric attenuation here are small since the distances which concern us are less than a meter or two and the frequencies are only 30 to 40 $\mathrm{kHz}$.

The bat's threshold for detecting sonar echoes declines by an estimated $11 \mathrm{~dB}$ for every doubling of target range, while the strength of the echoes themselves declines by $12 \mathrm{~dB}$ for every doubling of range. For target ranges from $17 \mathrm{~cm}$ out to 1 to 1.7 $\mathrm{m}$, the sensitivity of the bat's hearing for sonar echoes changes by an amount that approximately compensates for associated changes in the amplitude of the echoes themselves. Over this span of ranges, the bat holds its threshold at a constant level with respect to echoes returning from a small target. Echoes thus are heard at a constant sensation level. The solid line marked point target in Figure 3 traces the decline in echo sound pressure for echoes from a small target at progressively greater distances. This line has a slope of $-12 \mathrm{~dB}$ for every doubling of target range, plus a slight downward curvature due to the cumulative effect of atmospheric attenuation at frequencies of 30 to $40 \mathrm{kHz}$. Only the slope of the point target line concerns us here; lines with this same slope can be drawn at any vertical position on the graph depending upon the actual size of the object acting as a point target. Larger spherical targets reflect stronger echoes at any particular distance, but the decline in echo strength for a given target at different distances follows the slope of the curve for point targets in Figure 3 . The particular curve in Figure 3 shows how the echoes from a spherical target, which are just barely detectable by the bat at a distance of $17 \mathrm{~cm}$, would remain just barely detectable by the bat at greater and greater distances out to 1 to $1.7 \mathrm{~m}$, where the bat's hearing thresholds level off at about $0 \mathrm{~dB}$ SPL.

As a sonar target appears at shorter distances, or as the bat flies closer to the target, it receives increasingly intense echoes, but these echoes would remain at a fixed level with respect to the bat's hearing threshold even though the distance becomes smaller. The bat evidently possesses some mechanism within its auditory system which uses the time delay of echoes to compensate for echo intensity changes by changing the sensitivity of hearing. Such a mechanism functions as an automatic gain control for the bat's sonar receiver. Range-related control of sensitivity to echoes previously has been suggested to exist in echolocation (Johnson and Titlebaum, 1976), and its discovery in the data shown in Figure 3 reveals what must be an extremely important aspect of echo information processing. The shaping of the time course of sensitivity of hearing after the emission of a sonar signal to conform to the relative strength of echoes from a target at different distances indicates how thoroughly the bat's auditory system must be adapted for use as a sonar receiver.

The middle ear muscles and range-related gain control. Two experimental studies document the time course of the effects of middle ear muscle contractions upon the bat's sensitivity to sounds occurring shortly after vocalization (Henson, 1965; Suga and Jen, 1975). These muscles are at the most peripheral site in the bat's auditory system to consider as an obvious possibility for controlling sensitivity to echoes (Henson, 1967; Johnson and Titlebaum, 1976) because they contract at the time of emission of echolocation sounds and relax subsequently. In the two species of bats studied, Tadarida brasiliensis and Myotis lucifugus, the middle ear muscles begin to contract before any sound is produced and reach a maximum state of contractionand, therefore, of middle ear attenuation of sound-just at the time the sound is emitted. Measurements in $M$. lucifugus, for example, show that ultrasonic sounds reaching the cochlea of the inner ear through the middle ear are attenuated by 25 to $27 \mathrm{~dB}$ immediately after the emission. An interval of 5 to 8 msec is required for this attenuation to decay to zero as the muscles relax (Suga and Jen, 1975).

The maximum amount of attenuation produced by the middle ear muscles of bats corresponds very closely to the threshold elevation of $28 \mathrm{~dB}$ (from $8 \mathrm{~dB}$ SPL at $110 \mathrm{~cm}$ to $36 \mathrm{~dB}$ SPL at $17 \mathrm{~cm}$ ) observed in echo detection experiments (Fig. 3). Furthermore, the 5- to 8-msec time course of the decay of middle ear muscle contractions matches the time course of the threshold shift occurring afler emissions. It would therefore appear that most, if not all, of the rise in the bat's threshold for detecting echoes can be accounted for in terms of middle ear muscle contractions occurring in synchrony with emission of sonar sounds, assuming that the middle ear muscle observations in $T$. brasiliensis and $M$. lucifugus would also be true in $E$. fuscus.

The contraction of middle ear muscles in bats has been assigned a role in protecting the bat's hearing from the intense sonar vocalizations (Hartridge, 1945; Griffin, 1958; Wever and Vernon, 1961; Henson, 1965,1967; Suga and Jen, 1975). In addition, it has been suggested that their function extends beyond mere protection to include regulation of signal amplitudes to particular levels for optimal auditory processing (Suga 
and Jen, 1975). The correspondence between echo attenuation brought about by contractions of the middle ear muscles and the echo detection threshold shift shown in Figure 3 demonstrates that the middle ear muscles do in fact exercise a regulatory effect, matching sensitivity for echoes to the physical acoustics of the scattering of ultrasonic echoes from point targets at different distances from the bat. The concept of "regulation" implies a criterion to which something is adjusted. The role of the bat's middle ear muscles in compensating for the decrease in echo amplitude associated with increasing target range may thus be the first quantitatively complete example of such a regulatory function for the middle ear system. The fact that the regulatory action is exerted at the middle ear and, therefore, affects signals reaching the cochlea to stimulate the auditory nerve suggests that this regulation may occur for the benefit of the auditory nerve's responses to echoes.

A three-dimensional spatial tracking filter in echolocation. The discovery that $E$. fuscus apparently regulates the amplitude of echoes from targets at different distances to a stable amplitude at the inner ear sensitizes us to think about whether there are other mechanisms of this type in the bat's sonar receiver. The bat's middle ear muscle contractions appear to remove from echoes reaching the cochlea those variations in amplitude which are produced by changes in target range. Are there other regulatory actions which similarly eliminate echo intensity variations originating in changes in a target's horizontal and vertical position? The data in Figure 3 show that, when the bat is flying toward a target, echoes from the target probably fall under the influence of the middle ear automatic gain control at a distance of 1 to $1.7 \mathrm{~m}$. This distance corresponds roughly to the distance of 1 to $2 \mathrm{~m}$ at which an echolocating bat first shows overt reactions to the presence of a target such as a flying insect (Griffin, 1958; Griffin et al., 1960). These reactions have been considered as the end of the search stage of the process of intercepting prey and the start of the approach stage. As the bat is closing in to capture a target, it increases the rate of emission of its sonar sounds during the approach stage, and it also commences to track the target in horizontal and vertical directions by aiming its head at the target. As Figure 3 reveals, these two reactions may very well be accompanied by activation of the middle ear automatic gain control.

The bat emits sonar sounds that are moderately directional (Griffin, 1958; Pye, 1980), so that the strength of the sound which is incident upon the target depends upon whether the target is directly in front of the bat's mouth (or nasal emitter) or off to one side. The bat's action of pointing its head at the target keeps the target in the center of the beam of sound. The accuracy of the bat's head aim during interception has been judged roughly from stroboscopic photographs to be about $\pm 5^{\circ}$ (Webster and Brazier, 1965). Results obtained in recent experiments using new techniques with $E$. fuscus indicate that the accuracy of head aim tracking is more like $\pm 1^{\circ}$ (W. M. Masters, A. J. M. Moffat, and J. A. Simmons, manuscript in preparation). $E$. fuscus emits sonar sounds that have a directional beam width of about $\pm 22^{\circ}$ at $30 \mathrm{kHz}$ (Simmons, 1969). If the bat can track the target with an accuracy of even a few degrees, the sound that is incident upon the target will probably vary by only $1 \mathrm{~dB}$ or so due to directional effects. The amount of variation in the vertical plane is likely to be similar (Shimozawa et al., 1974).

The bat's hearing, too, is directional (Henson, 1970; Grinnell and Schnitzler, 1977; Schnitzler and Henson, 1980). Sounds coming from straight ahead or slightly to one side are received through the external ear, which acts as a receiving antenna, with greater sensilivily than sounds coming from directions more extremely to the side, above, or below (Grinnell and Grinnell, 1965). The directionality of sound reception is only moderately sharp in $E$. fuscus, whether measured physiologically (N. Suga, personal communication) or acoustically (J. A.
Simmons, manuscript in preparation). Again, if the bat keeps its head pointed at the target with an accuracy of several degrees, variations in the strength of sounds from straight ahead reaching the eardrum through the external ear will be about $1 \mathrm{~dB}$. When both sound emission and reception are taken into account, echoes reaching the bat's ears from a target that is within a few degrees of straight ahead will only vary by 1 or $2 \mathrm{~dB}$ due to directional effects (J. A. Simmons, manuscript in preparation). In contrast, if the target were $10^{\circ}$ to one side, echoes would be $4 \mathrm{~dB}$ weaker at the eardrum, and, if the target were $30^{\circ}$ to one side, echoes would be 10 to $15 \mathrm{~dB}$ weaker.

It should be noted that this discussion of directonality concerns changes that would occur in echo strength at the eardrum if the target were to wander off of the main axis of the echolocation system. Directionality as such is only peripherally related to the bat's actual perception of the target's direction, which for $E$. fuscus appears to be based primarily upon the timing of echoes at the two ears for horizontal directions (Simmons et al., 1983) and the fine structure of echo waveforms reverberating through the complex directional transfer function of the external ears for vertical directions (Lawrence and Simmons, 1982b). (Whether these echo timing cues are mediated within the auditory system by time or frequency domain representations is irrelevant to this point.) The overall intensity of echoes may not be used by $E$. fuscus for fine localization but instead is kept relatively stable at the two ears by the bat's tracking of the target with head aim, a response that we suggest may be driven from perception of target position based on echo timing cues.

Clearly, one effect of the bat's reaction of keeping its head pointed at the target would be to stabilize echo amplitude at the eardrum and, therefore, at the inner ear, after the target is detected. Variations in echo amplitude that would occur as the target moves in different directions relative to the body axis or flight velocity vector of the bat are reduced because the bat tracks the target with its head and ears. In conjunction with the middle ear muscle contractions, the bat's head movements can be seen as part of a system which prevents echoes reaching the bat's cochlea from varying greatly in amplitude due to the changing momentary position of the target relative to the bat. We propose that echolocating bats such as $E$. fuscus possess a spatial tracking filter which locks onto the target's range, horizontal direction, and vertical direction, tracking the target as it moves relatively nearer and from one horizontal or vertical position to another during pursuit. The immediate effect of this three-dimensional spatial tracking filter is to nullify echo amplitude variations related to the target's spatial position with respect to the bat.

Interception of flying insects by echolocation. Bats use their sonar to detect, locate, and identify the flying insects which they feed upon. The pursuit of prey is a relatively stereotyped sequence of behaviors in many species of bats (Griffin, 1958; Ajrapetjantz and Konstantinov, 1974; Novick, 1977; Simmons et al., 1979b; Schnitzler and Henson, 1980) and we can best discern the significance of the data shown in Figure 3 if we place it in the context of interception of prey. There are three distinct stages to the acoustic behavior of bats during the interception process-the search, approach, and terminal stages-which are identified entirely by the pattern of emission of sonar sounds. When a bat has approached to a distance of 1 to $1.5 \mathrm{~m}$ from a flying insect or an airborne target, it begins to increase the repetition rate of its sonar signals from approximately 10 to 20 sounds/sec to approximately 30 to 50 sounds/ sec (Griffin, 1958; Griffin et al., 1960; Webster and Brazier, 1965). This event marks the transition from the search stage to the approach stage. When the bat has approached to about 20 to $50 \mathrm{~cm}$ from the target, it abruptly increases the repetition rate of its emissions to as much as 100 to 200 sounds/sec. The second, especially dramatic increase marks the transition from 
the approach to the terminal stage. Shortly thereafter, the bat seizes the target in its wing or tail membrane (Webster and Griffin, 1962). The terminal stage is often called the "feeding buzz" because the rapid sequence of sonar emissions makes a buzzing sound when listened to with a "bat detector" (Griffin, 1958; Sales and Pye, 1974; Simmons et al., 1979a). The search stage, beginning at a distance of 1 to $1.5 \mathrm{~m}$, really refers to a period prior to the bat's first reliable acoustic reaction to the target's presence, rather than to a period prior to detection itself (Griffin, 1958). Eptesicus can detect individual insectsized spheres at greater distances of 3 to $5 \mathrm{~m}$ (Kick, 1982), and there are also some observations of bats in flight reacting to such targets at distances of several meters (Griffin, 1958; Webster and Brazier, 1965; Ajrapetjantz and Konstantinov, 1974).

The sequence of perceptual events taking place simultaneously with the acoustical stages shown by the bat's emissions are variously described as target detection, localization, trajectory evaluation, target discrimination, and capture (Webster, 1967), or detection, fixation, tracking, and capture (Schnitzler and Henson, 1980). Eptesicus can detect insect-sized targets at distances of 3 to $5 \mathrm{~m}$ (Kick, 1982), although it is not known whether flying bats might integrate information across several echoes to decide that a target is present. If so, the detection of a flying insect might occur a little nearer than detection of a sphere. In addition, at about the time of the noticeable increase in repetition rate marking the beginning of the approach stage, the bat begins to track the direction of the target with the aim of its head (Webster and Brazier, 1965; Webster, 1967). Localization or fixation must therefore have occurred by this time. Furthermore, experiments in which bats choose between a mealworm and a plastic or metal target thrown into the air reveal that the bat makes its decision identifying the target as edible or inedible from echoes received prior to the terminal stage. Thus, bats use echolocation to discriminate targets by size and shape, probably when they are between about 1.5 and $0.5 \mathrm{~m}$ away, or during the approach stage (Griffin et al., 1965). We see from Figure 3 that, when the bat is increasing the repetition rate of its sonar signals, tracking the target with the aim of its head, and attempting to identify the target, it also appears to experience range-related changes in hearing sensitivity caused by the middle ear gain control system. Because the acoustic rather than the perceptual stages of interception are most frequently used in describing the bat's behavior, it is desirable to modify the three-stage description to include a new stage which explicitly recognizes the bat's tracking activities.

The four stages of interception of prey. Figure 4 shows a composite diagram of the capture of an insect by an aerialfeeding bat (Fenton, 1982) which emits predominantly FM

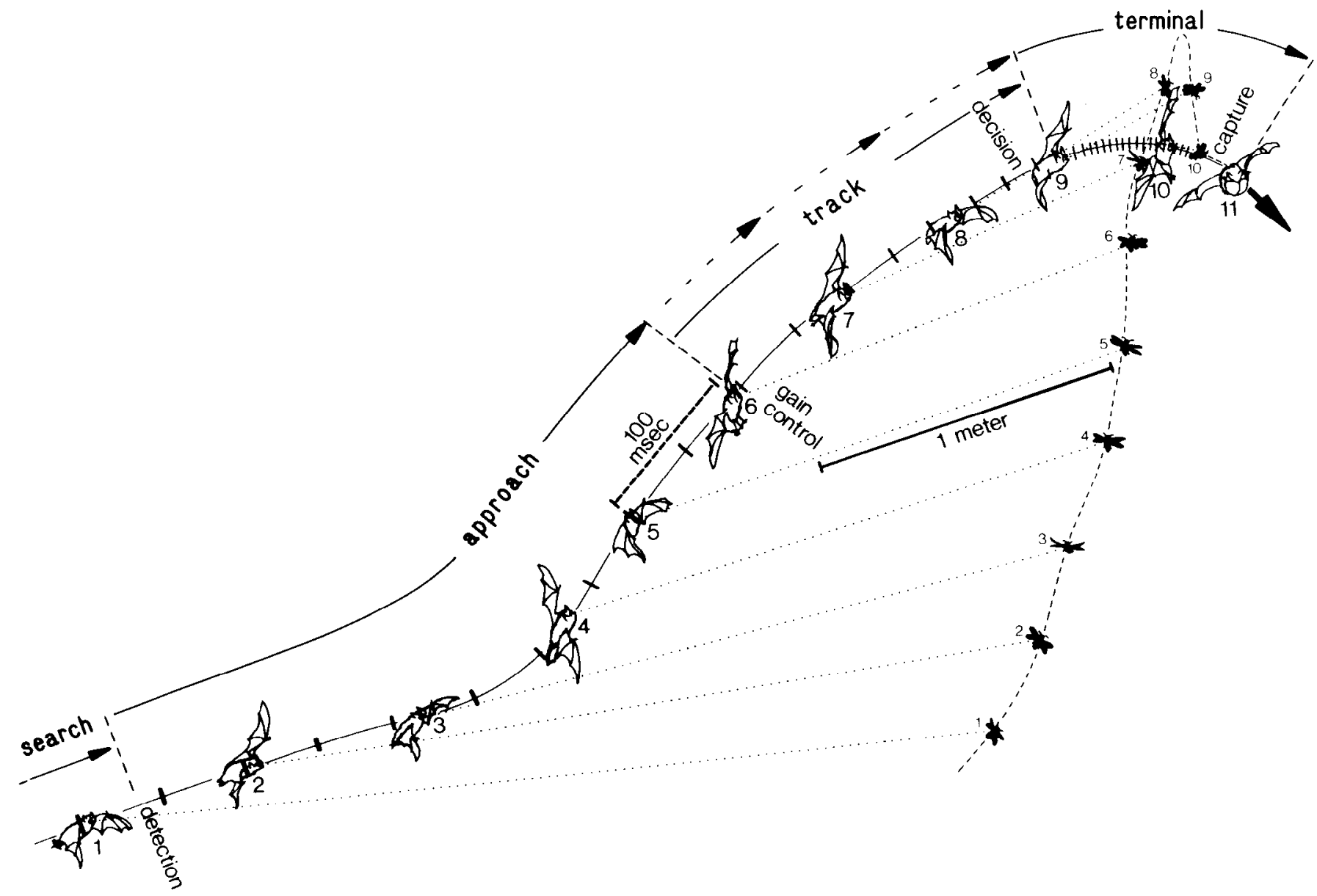

Figure 4. A diagram of successive positions of an insectivorous bat and a flying insect during the bat's interception of the insect (based on stroboscopic photographs by Webster (1967)). The images of the bat ( 1 to 11 ) and of the insect ( 1 to 10) are separated by $100 \mathrm{msec}$. The distance from the bat to the insect for each pair of images appears as a dotted line. The bat's sonar emissions appear as short bars perpendicular to the bat's flight path. The bat detects the target (between images 1 and 2 ) at a distance of $3 \mathrm{~m}$, thus ending the search stage of pursuit. The bat flies nearer to the target during the approach stage, while receiving echoes that progressively increase in strength (images 2 to 6 ). At a distance of 1.3 $m$ (image 6) the bat enters the region in which its echo gain control functions, and it increases the rate of emission of its sonar sounds while also tracking the target's position with the aim of its head and ears. The bat in the tracking stage (images 6 to 9 ) is observing target features to decide whether it is an insect and worth capturing. The terminal stage (images 9 to 11) involves the bat in coordinating its flight to seize the insect in its wing or tail membrane and is characterized by a very high rate of emission of sonar sounds. 
sonar sounds, such as $M$. lucifugus or $E$. fuscus. It is based upon stroboscopic photographs of an actual interception made by $M$. lucifugus (Webster, 1967) combined with drawings of hypothetical actions immediately preceding the photographs. In Figure 4, the bat (image 1, on the left) initially detects the target, which is a small flying insect (image 1, on the right), at a distance of $3 \mathrm{~m}$. The drawing shows 11 successive images of the bat and the target separated in time by $100 \mathrm{msec}$, the rate at which the stroboscopic flash operated in obtaining the actual photographs of images 7 to 11 . The images from 1 to 6 are drawn from and are entirely in keeping with the previous photographic studies of interception (Griffin, 1958; Webster and Brazier, 1965; Webster, 1967). In addition to the images of the hat and the insect, the diagram shows schematically the time of occurrence of echolocation sounds (as short bars placed perpendicular to the solid line tracing the bat's flight path) and the four stages in the pursuit process, indicated as search, approach, track, and terminal.

At a distance of about $3 \mathrm{~m}, E$. fuscus is just able to detect a spherical target with a diameter of $5 \mathrm{~mm}$ (Kick, 1982). Prior to detection (Fig. 4, image 1) the bat would have been flying in the search stage of pursuit, emitling sonar sounds at a rate of about 10 to $20 / \mathrm{sec}$, and presumably would have been vigilantly seeking a target. After detection, the bat gives little sign that it has detected the target (Fig. 4, images 2 to 5) until it has approached to within approximately $1.5 \mathrm{~m}$ (Fig. 4, image 6). Even so, during this interval the bat is receiving echoes from the target and must be perceiving the target to some extent. This is the approach stage as we shall consider it here. At a distance of about $1.3 \mathrm{~m}$ in this example (Fig. 4, image 6), the bat begins to react overtly to the target by gradually increasing the repetition rate of its sonar emissions to about 40 sounds/ sec and aiming its head to track the target (Griffin, 1958; Webster and Brazier, 1965). The sonar sounds also become shorter, and approximately at this distance the bat's middle ear gain control starts to regulate the amplitude of echoes from the insect to a fixed level above the bat's threshold (see Fig. 3). This is the beginning of the tracking stage of pursuit, during which the bat locks onto the target in range, horizontal direction, and vertical direction using the three-dimensional spatial tracking filter. During the tracking stage of interception (images 6 to 9 in Fig. 4), echoes reaching the bat's cochlea will vary significantly in amplitude only if the target's acoustic reflectivity changes from one moment to the next or if the bat changes the intensity of its emissions. The tracking filter cancels out the larger part of echo amplitude variability which originates in the target's changing position with respect to the bat. Bats trained to discriminate between edible and inedible airborne targets make the decision concerning whether to capture the target during the tracking stage (Griffin et al., 1965). When the bat has flown to within 20 to $50 \mathrm{~cm}$ of the insect (Fig. 4, image 9), it enters the terminal stage of pursuit and completes the capture of the target by seizing it in the wing or tail membrane (Webster and Griffin, 1962). If the bat decides not to capture the target because it judges the target to be an inedible object, it breaks off the pursuit by failing to enter the terminal stage.

Stimulation of the bat's ears during interception. The sonar signals transmitted by insectivorous bats which forage in the air for flying insects are exceedingly intense. Signals recorded in the laboratory at distances of about $10 \mathrm{~cm}$ from the bat commonly have peak-to-peak amplitudes of 100 to $110 \mathrm{~dB}$ SPL ( $\mathrm{dB}$ re $20 \mu \mathrm{Pa}$ ), and similar magnitudes are estimated from recordings made under field conditions (Griffin, 1958; Novick, 1977; Pye, 1980). From comparisons of laboratory and field recordings of the echolocation signals of $E$. fuscus (Griffin, 1958; Simmons, 1979; Kick, 1982), we estimate the sonar signals likely to be emitted as the bat flies through the search, approach, and tracking stages of Figure 4 at about 109 to $110 \mathrm{~dB}$
SPL peak to peak. During the terminal stage of pursuit, the bat progressively decreases the strength of its emissions by a total of about $6 \mathrm{~dB}$ (Griffin, 1958; Webster, 1967). The level of signals observed during interception also varies in an irregular fashion by several decibels, but we assume this to be a consequence of the bat's motion relative to the microphone since signals recorded in the laboratory when the bat's head is stationary do not vary much at all (Simmons and Vernon, 1971 ). When the sonar emissions of $E$. fuscus have peak-topeak amplitudes of 109 to $110 \mathrm{~dB}$ SPL, the sound pressure at $30 \mathrm{kHz}$ is about $104 \mathrm{~dB}$ SPL (Kick, 1982). We thus know approximately the strength of the signals which the bat transmits at the target. Furthermore, we know that these sonar signals have an effective strength at the cochlea that is 40 to $50 \mathrm{~dB}$ weaker: 20 to $25 \mathrm{~dB}$ of this is due to the directional beaming properties of the emissions and the directional receiving properties of the ears, and the rest is due to the synchronized contractions of the middle ear muscles (Henson, 1967, 1970; Suga and Jen, 1975; Jen, 1982; J. A. Simmons, manuscript in preparation; N. Suga, personal communication).

Figure 5 shows the estimated sound pressures of the sonar signals enitted by the bat and the echoes received from the target during the interception illustrated in Figure 4. (See the legend to Fig. 5 for an explanation of its relationship to Fig. 4). Figure $5 A$ shows the estimated amplitude of the sonar emissions which were depicted in Figure 4 as short bars placed along the bat's flight path. The values given are for peak-to-peak sound pressure at approximately $10 \mathrm{~cm}$ from the bat's mouth and at a frequency of $30 \mathrm{kHz}$ in the FM sweep emitted by the bat. These emissions have ampitudes 40 to $50 \mathrm{~dB}$ lower at the cochlea. Thus, the bat hears its own sonar signals at about 54 to $64 \mathrm{~dB}$ SPL during the search, approach, and tracking stages of pursuit, with amplitudes falling to about 48 to $58 \mathrm{~dB}$ during the terminal stage. The middle ear muscles of bats do not continue to contract synchronously with vocalizations at the highest repetition rates of the terminal stage. Instead, they reach a condition of tetanic contraction, resulting in attenuation of emissions and echoes alike (Henson, 1965; Suga and Jen, 1975). Since echoes return at very short delays during the terminal stage, the effects of the gain control system probably are preserved into the terminal stage through the combination of reduced amplitudes of emissions and sustained contractions of middle ear muscles.

Figure $5 B$ shows the peak-to-peak amplitude of echoes reflected back to the bat if the target in Figure 4 is a sphere with a diameter of $5 \mathrm{~mm}$. Echoes from the sphere are $35 \mathrm{~dB}$ weaker at $30 \mathrm{kHz}$ just due to the acoustic characteristics of the target (Griffin, 1958; Kick, 1982). The solid line (solid data points) in Fig. $5 B$ shows the progressive increase in echo strength from the spherical target as the bat flies nearer during the interception maneuver. The dashed line (open data points) in Figure $5 B$ shows the amplitude of echoes from a target with the same 35 $\mathrm{dB}$ average echo reduction as the $5-\mathrm{mm}$ sphere, but with a \pm 5 $\mathrm{dB}$ amplitude modulation at a rate of $30 \mathrm{~Hz}$ to simulate changes in echo strength due to the target's tumbling motions in the air or to such actions as insect wing beats. This is a reasonable rate and amount of modulation; airborne mealworms and other experimental targets reflect echoes that vary over this range from one aspect angle to another within the time available for the bat to gather echoes (Griffin, 1967), and echo amplitude modulations from flying moths match or even exceed this range depending upon the aspect angle and the size of the moth (Schnitzler, 1983).

The curves in Figure $5 B$ show the manner in which stimuli reaching the bat's external ears change throughout the pursuit of prey. The bat would detect the $5-\mathrm{mm}$ target at a distance of $3 \mathrm{~m}$ when the amplitude of echoes first rises above the longrange detection threshold in the region of $0 \mathrm{~dB} S \mathrm{SL}$, thus 


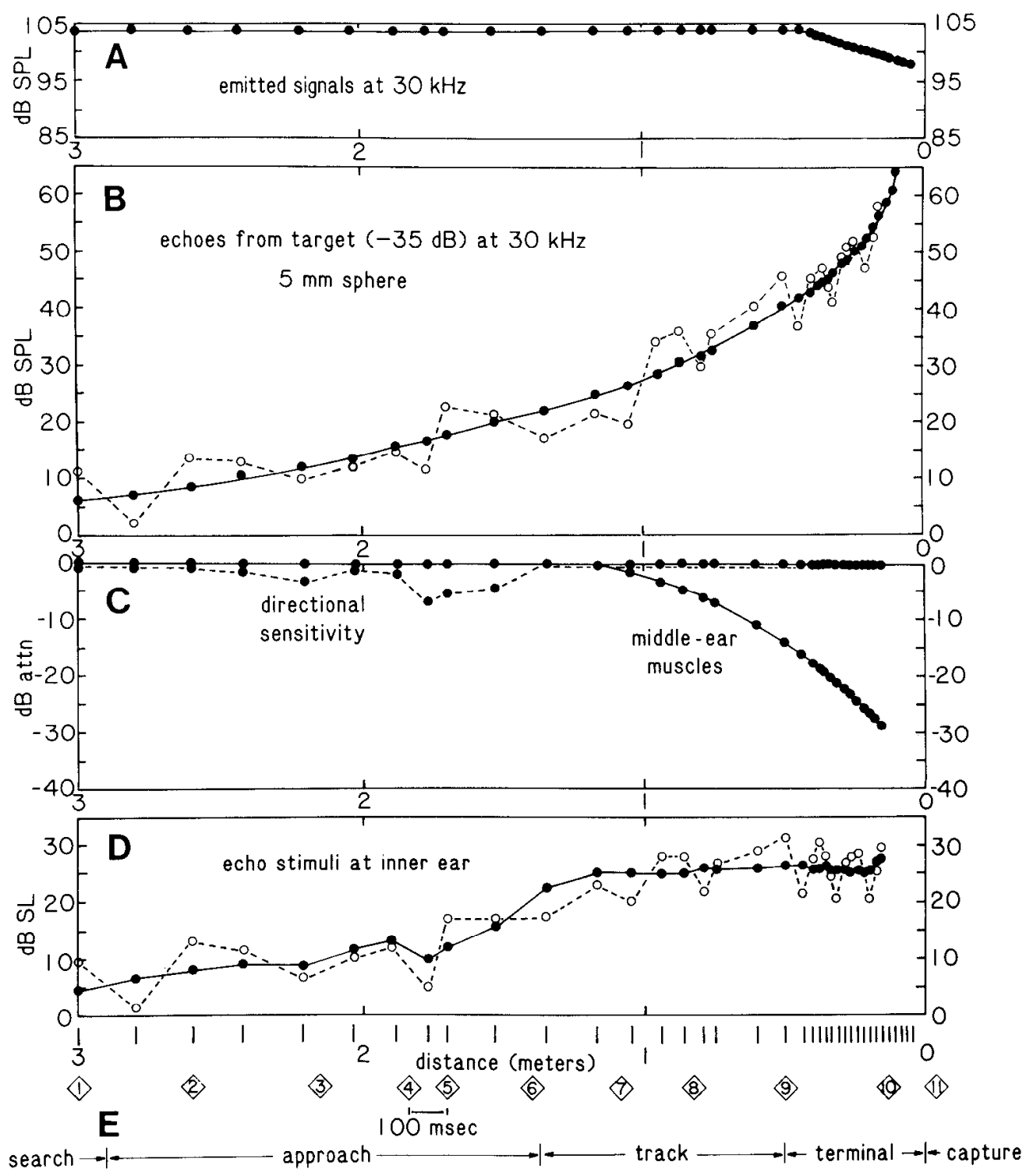

Figure 5. Graphs for determining the level of stimulation reaching the bat's inner ear during the interception shown in Figure 4. 'The horizontal axis gives the distance from the bat to the target, and successive image numbers from Figure 4 are shown in diamonds along the horizontal axis. Vertical bars along the horizontal axis show the time (or position) of the bat's sonar signals. The stages of pursuit are indicated at the bottom $(E)$. $A$ gives the peak-to-peak sound pressure of the bat's sonar emissions at 30 $\mathrm{kHz} . B$ shows the strength of the echoes returning from the target. The solid curve shows echoes from a $5-\mathrm{mm}$ sphere, and the dashed curve shows echoes from a model insect with $30-\mathrm{Hz}$ wing beats and $\pm 5 \mathrm{~dB}$ echo amplitude modulation. $C$ shows the effects of the bat's spatial tracking filter, consisting of the middle ear response (solid curve) and the head aim response (dashed curve). $D$ shows the estimated strength of echoes at the bat's cochlea from the 5-mm sphere (solid curve) and from the "insect" (dashed curve). Note that the vertical axis for $D$ is in terms of echo sensation level, or sound pressure with respect to the bat's threshold.

ending the search stage. As the bat flies nearer, the echoes increase gradually in amplitude to a level about 20 to $25 \mathrm{~dB}$ above threshold, at a distance of $1 \mathrm{~m}$. The amplitude of echoes then rises more steeply as the bat approaches nearer yet, with sound pressures of about $60 \mathrm{~dB}$ SPL toward the end of the terminal stage. At the bat's eardrum, the strength of stimulation directly caused hy the emissions (approximately 20 to $25 \mathrm{~dB}$ weaker than the emissions, or 73 to $78 \mathrm{~dB}$ SPL) is not much greater than the level of stimulation produced by echoes from an insect-sized target in the final moments of pursuit. Larger targets actually yield echoes at close range that are stronger than stimulation provided by the emissions themselves (Henson, 1967, 1970). Amplitude modulations imposed upon echoes by the beating of the "insect's" wings are very noticeable as variations in the course of the dashed line around the smooth curve followed by the solid line in Figure $5 B$.

Figure $5 C$ shows the combination of the effects of the gradual relaxation of the bat's middle ear muscles following their contraction synchronous with vocalization, and the tracking of target direction with the aim of the bat's head. Echoes reaching the bat's middle ear are stripped of amplitude variations related to target direction by the head aim tracking response, which commences in Figure 4 approximately at image 6. Echoes reaching the bat's inner ear additionally are stripped of amplitude variations related to target range by the state of relaxation of middle ear muscles. The dashed curve in Figure $5 \mathrm{C}$ in 
particular shows the amount of attenuation of echoes stimulating the bat's cochlea as a consequence of the misalignment of the bat's head aim with the target's direction between images 3 and 6 in Figure 4. (The bat's head is assumed to point straight ahead, along the direction of flight, until the bat begins its tracking response at image 6). The solid curve in figure $5 \mathrm{C}$ shows the amount of echo attenuation accumulated by the middle ear muscle contractions as the bat moves closer than 1 $\mathrm{m}$ to the target and the delay of echoes falls below $6 \mathrm{msec}$. The regression line from Figure 3 is plotted as the middle ear muscle curve in Figure 5, with middle ear attenuation starting to weaken echoes at a range of $1.1 \mathrm{~m}$. This is conservative, since the low thresholds for echo detection estimated from experiments with 4.8 - and 19.1 -mm spheres suggest that, at a distance of $1.1 \mathrm{~m}$, about $8 \mathrm{~dB}$ of middle ear muscle attenuation may already be present.

Figure $5 D$ shows the amplitude of echoes from the target actually reaching the bat's inner ear to stimulate the cochlea. The solid curve (solid data points) in Figure $5 \mathrm{D}$ gives echo amplitudes above the bat's threshold (sensation levels) for echoes from the 5- $\mathrm{mm}$ sphere, and the dashed curve (open data points) gives echo amplitudes from the "insect." These curves demonstrate dramatically the consequence of the action of the bat's spatial tracking filter in stabilizing the amplitude of echoes for the bat's auditory nervous system to process. Echoes from insect-sized spherical targets are regulated to a steady, rather low sensation level during the tracking and terminal stages of pursuit. Because amplitude variations related to the target's location are effectively eliminated, those variations related to the target's fluttering movements become very conspicuous. It seems likely that one important function of the tracking filter system is to render target-related variations in echo strength more readily available to the bat's perception than they would be if combined with the much larger variations originating in the target's changing range and direction. The overall size of the target is still available for the bat to perceive from the average sensation level of echoes during the tracking stage of pursuit, since larger targets will yield echoes that stimulate the cochlea more strongly. The size of a target is an important cue for the bat to use in distinguishing targets, and fluctuations in echo amplitude from moment to moment also appear important for the bat to make more subtle distinctions (Griffin et al., 1965; Griffin, 1967; Simmons and Vernon, 1971; Neuweiler et al., 1980; Schnitzler and Henson, 1980).

The definitions of the four proposed acoustic stages of pursuit shown in Figures 4 and $5 E$ are apparent from the curves in Figure $5(A$ to $D)$. The original definitions of the search and terminal stages refer, respectively, to the bat not giving any sign of having detected the target, and to the bat's energetic efforts to intercept and seize the target in the final instant of the whole maneuver (Griffin, 1958; Griffin et al., 1960). These two definitions seem obvious and straightforward. Now that we know that the bat initially detects insect-sized targets at longer ranges than 1 to $1.5 \mathrm{~m}$ (Kick, 1982), we ought to move the search stage to an earlier period which ends with the reception of the first echoes from the target above the threshold for echo detection. The search stage thus would end at a distance which depends upon the target's size and the relationship of its echoes to the data shown in Figure 3. Large insects would be detected at $5 \mathrm{~m}$ or more, medium-sized insects at distances of 2 to $4 \mathrm{~m}$, and small insects at distances of 1 to $2 \mathrm{~m}$. There appears to be a minimum size to the targets which the bat can detect in the pursuit process. The bat's rising threshold for detecting earlier echoes will keep targets that are below threshold at $1 \mathrm{~m}$ below threshold at shorter ranges, too. Individual spherical targets as small as $1 \mathrm{~mm}$ in diameter would thus never be detected by $E$. fuscus. Clusters of such small targets might be detected, however, since the aggregate echo ought to exceed the bat's thresh- old as the bat flies nearer. Myotis can in fact capture spherical targets as small as $1.6 \mathrm{~mm}$ in diameter from airborne clusters (Webster and Brazier, 1965; Webster, 1967), but smaller targets appear not to be detected.

The original definition of the approach stage of pursuit was based upon the overt responses which bats consistently make to the presence of a target when they reach a distance of roughly $1.5 \mathrm{~m}$ (Griffin, 1958; Griffin et al., 1960). The data shown in Figure 3 and the curves in Figure 5 make it clear that the bat's activities beginning at a distance of about $1.5 \mathrm{~m}$ are very special. The bat begins to track the target in range with its middle ear muscle contractions, in horizontal and vertical direction with its head aim response, and in terms of its acoustic images by changing the characteristics of its sonar signals (Simmons et al., 1979b). The approach stage here describes the bat's behavior after the target is detected but before any of these tracking mechanisms come into operation at a range of about $1.5 \mathrm{~m}$.

A flying insect beats its wings and twists and turns in the air, offering to the bat relatively large changes in its acoustic reflective characteristics from one echo to the next (Griffin et al., 1965; Griffin, 1967; Schnitzler, 1983). Once the target is detected, the bat would have the opportunity to observe these target-related changes in amplitude from one echo to another. This is particularly true for the tracking stage because other, larger changes in amplitude are removed by the automatic gain control and tracking filter system. Echo-to-echo amplitude variations in different frequency bands also may be an important cue for the bat to use in discriminating the shape of airborne targets (Griffin, 1967). The bat may wait until the target is within $1.5 \mathrm{~m}$ or so, and the gain control is active, to begin seriously examining the target to identify it, and we know that the bat probably makes decisions concerning the target during the tracking stage (Griffin et al., 1965). The bat's deferred reaction to the target, from detection at several meters to overt reaction at roughly 1 to $1.5 \mathrm{~m}$ (Griffin, 1958; Schnitzler and Henson, 1980; Kick, 1982), may represent a range gating mechanism linked to activation of the gain control mechanism. The consistent triggering of the tracking stage's characteristic behavior pattern at a distance of about $1.5 \mathrm{~m}$ seems related to target range rather than to target detection now that we know detection to occur substantially earlier.

During the approach stage the bat receives a series of echoes which increase progressively in amplitude from the region of threshold at $0 \mathrm{~dB}$ SPL to a level of about $20 \mathrm{~dB}$ above threshold. Substantial further increases do not become manifest at the inner ear due to the gain control system coming into effect during the tracking stage. Most of the approach stage thus is characterized by reception of echoes at such low sensation levels that the bat may form only an imprecise acoustic image of the target. Furthermore, in some species of bats the signals emitted during this approach stage are still essentially detection signals; they are narrow in bandwidth (Simmons et al., 1979b) and cannot provide the bat with much detailed information about the target. Only during the tracking stage do these bats begin to use sonar signals of broader bandwidth that can convey precise information about a target's location, and only during the tracking stage will echoes of these signals probably have sufficient amplitude relative to threshold for the bat to extract accurate information about the target's location. The approach stage would be shorter for smaller targets, since these would be detected at closer range, while the end of the approach stage is fixed by the stereotyped reactions that mark the tracking stage at a distance of about $1.5 \mathrm{~m}$. Spherical targets with diameters smaller than $3 \mathrm{~mm}$ would evoke the reactions of the tracking stage only after the bat has approached nearer than $1.5 \mathrm{~m}$; detection would take place so close to the target that the tracking response would begin at once. In these conditions the perceptual qualities of the approach and tracking stages over- 
lap, as indicated in Figure 4 by the arrows extending the approach stage past image 7 .

Physiological consequences of stabilization of echo amplitude. Stabilization of the amplitude of stimulation of the cochlea by echoes has two extremely important consequences for neural activity evoked by echoes in the auditory nerve. Bats perceive the distance to a target from the arrival time of echoes (Simmons, 1973), and the limiting accuracy with which bats perceive echo arrival time is partly determined by the acoustic waveform of the sonar signals and echoes (Simmons, 1979) and probably also by the signal-to-noise ratio of echoes (Skolnik, 1962; Woodward, 1964; Schnitzler and Henson, 1980). Neurophysiological experiments indicate that the representation of the time of occurrence of FM signals such as echolocation sounds in the most peripheral parts of the auditory nervous system is in terms of the time of occurrence of nerve discharges in fibers tuned to individual frequencies occurring in the FM sweep (Suga, 1973; Pollak et al., 1977). The latency of discharges for neurons in the auditory system is dependent upon stimulus amplitude; changes in the amplitude of echoes stimulating the bat's cochlea produce shifts in the timing of nerve impulses. A change of 30 to $40 \mathrm{~dB}$ in echo amplitude can produce a shift in the timing of nerve discharges of several milliseconds, and even the smallest shifts observed are about $1 \mathrm{msec}$ (Suga, 1970; Pollak et al., 1977; Pollak, 1980). To the bat, 1 msec corresponds to a change of $17 \mathrm{~cm}$ in echo range, which is many times larger than the smallest shift in range which the bat can directly perceive (Simmons, 1973). In fact, $17 \mathrm{~cm}$ is the minimum range shift that neurophysiological data lead us to expect that a 30to 40-dB shift in echo amplitude would produce in the bat's perception of target range. Bats usually capture insects in the wing or tail membranes (Webster and Griffin, 1962), and they would certainly fail to do so quite frequently if their perception of the target's distance were perturbed by as much as $17 \mathrm{~cm}$. The regulation of echo amplitude at the cochlea to a stable level by the middle ear system would prevent large shifts in echo amplitude from appearing in the stimuli delivered to the cochlea while the bat approaches nearer to a target from an initial distance of 1 to $1.5 \mathrm{~m}$. Large intensity-related changes in neural response latency would thus not occur during the tracking stage.

Most of the insects pursued by bats such as $F$. fusrus are relatively small sonar targets--mayflies, June beetles, and small flies and moths are fairly representative of the sizes frequently captured (Griffin, 1958; Novick, 1977; Fenton, 1982). These insects have acoustic cross-sections roughly comparable to spheres with diameters of 0.2 to $2 \mathrm{~cm}$, although the insects will have quite different apparent sizes or cross-sections from different aspects (directions) and from moment to moment (Griffin et al., 1965; Griffin, 1967; Schnitzler, 1983). The intensities of echoes from such small targets during the tracking stage will probably range from about 0 to $35 \mathrm{~dB}$ SPL peak to peak (Griffin, 1958; Kick, 1982). From Figure 3, targets with echoes of this amplitude at distances of 1 to $1.5 \mathrm{~m}$ will therefore have echoes only 0 to $35 \mathrm{~dB}$ above the bat's threshold of hearing. Stimulation by echoes reaching the bat during the pursuit of prey would thus activate auditory nerve fibers only in the socalled "tip" region of the tuning curves for these fibers.

The most salient feature of the response of auditory nerve fibers in mammals to higher-frequency, brief sounds delivered at low intensities and at frequencies within the tip of the fiber's tuning curve is that the response usually consists of a single discharge which marks the time of occurrence of the onset of the sound (Kiang, 1965). At stimulus levels between 0 and perhaps $20 \mathrm{~dB}$ above the fiber's threshold there is not even an increase in the total number of discharges per unit time above the spontaneous firing rate, just a grouping in time or synchronization of the same number of discharges to the times of repeated stimulation (Johnson, 1980). At stimulus levels above the threshold for actually increasing the total number of discharges per unit time above the spontaneous level, usually there still will only be a single discharge marking the time of occurrence of a brief stimulus such as the very transient sweep of a bat's FM sound through the excitatory area of the tuning curve (Pollak et al., 1977). More than one discharge for each stimulus only occurs when such transients are strong enough to activate the neuron in regions of the tuning curve considerably elevated from the tip. As a result of all this, the auditory nerve of a bat approaching an insect most conspicuously will carry information about the time of occurrence of individual frequencies in the FM sweeps of echoes. Intensity information is unlikely to be richly represented by the total number of impulses in each fiber evoked as a burst of activity by each echo. It is more likely to be represented by the differing sensitivities of neurons tuned to the same frequency, with only the most sensitive neurons responding to the weakest echoes. Models of echolocation that are based upon spectrogram-like neural representations of acoustic waveforms seem most useful to pursue at this juncture (Suga, 1973; Altes, 1980; Simmons, 1980).

Internal noise and detection of echoes. The bat's threshold for detecting echoes is determined ultimately by the strength of echoes relative to noise (Skolnik, 1962; Woodward, 1964). This noise could be acoustic noise originating externally in the environment, or it could be noise originating within the bat's auditory system. Because external noise passes through the middle ear system along with echoes and is attenuated as well as echoes by contraction of the middle ear muscles, the data in Figure 3 show that the bat's ability to detect echoes (at least in the conditions of these experiments) is limited by internal noise, not external noise. The signal-to-noise ratio of echoes to external noise ought to remain unaffected by middle ear attenuation, whereas attenuation of echoes by the middle ear would weaken echoes with respect to noise manifested in the inner ear or the auditory nervous system. This internal noise seems unlikely to be of an acoustic nature since it would have to be ultrasonic in frequency; it may originate instead in the transduction processes of the inner ear-in the hair cells of the organ of Corti or in their synaptic connections with auditory nerve fibers.

During the approach stage, as the signal-to-noise or signalto-threshold ratio of echoes progressively increases from 0 to $25 \mathrm{~dB}$ (in the example shown in Fig. 5), the bat would become increasingly accurate in perceiving the time of occurrence of echoes (Schnitzler and Henson, 1980). Perceptions of target range, horizontal direction, and vertical direction, which may be based in part on temporal cues (Simmons, 1979; Lawrence and Simmons, 1982b; Simmons et al., 1983), would become increasingly acute as echoes increase in strength over the internal noise of the bat's sonar receiver. If the bat relies upon the timing of echoes and temporal information in echoes to perceive the target's location in three dimensions and the intensity of echoes to distinguish the target, the bat may effectively isolate even the cues for target location from the cues for target identity, using the spatial tracking filter to improve the quality of both kinds of information. The echo signal-to-noise ratio apparently becomes good enough during the tracking stage for the bat to enter the target's range on a neural map of distances in the auditory cortex (Suga and O'Neill, 1979). It is interesting that the neural map becomes most organized and well defined for targets at distances of 1.5 $\mathrm{m}$ and less, the zone within which echo gain control operates. Perhaps the range gate mechanism for initiating the tracking stage may be located in, or at least activated by, the $1.5-\mathrm{m}$ site on the cortical map.

The bat's spatial tracking filter system is composed of the external ear, the middle ear, and such behavioral responses as the aim of the bat's head and the changes in sonar emissions 
occurring during the tracking stage of pursuit. It has proven necessary for us to have the behavioral data shown in Figure 3 to discern the existence of the system, even though sufficient physiological data have been available for one or two decades. We could ask for no more satisfying a demonstration of the central role of ethological and behavioral research in the neurosciences, not merely to isolate and define problems which can then be approached with the modern technical arsenals of anatomy and physiology, but to tell us what the results of this approach will mean.

\section{References}

Ajrapetjantz, E. Sh., and A. I. Konstantinov (1974) Echolocation in Nature (English transl.), Joint Publications Research Service, Arlington, VA, No. 63328.

Altes, R. A. (1980) Detection, estimation and classification with spectrograms. J. Acoust. Soc. Am. 67: 1232-1246.

Fenton, M. B. (1982) Echolocation, insect hearing, and feeding ecology of insectivorous bats. In Ecology of Bats, T. H. Kunz, ed., pp. 261285, Plenum Press, New York.

Gallego-Juárez, J. A., and L. Gaete-Garretón (1983) Propagation of finite-amplitude ultrasonic waves in air. I. Spherically diverging waves in the free field. J. Acoust. Soc. Am. 73: 761-767.

Grant, D. A. (1946) New statistical criteria for learning and problem solution in experiments involving repeated trials. Psychol. Bull. 43: $272-282$.

Griffin, D. R. (1958) Listening in the Dark, Yale University Press, New Haven, CT.

Griffin, D. R. (1967) Discriminative echolocation in bats. In Animal Sonar Systems: Biology and Bionics, R. -G. Busnel, ed., Vol. I, pp. 273-299, Laboratoire de Physiologie Acoustique, Jouy-en-Josas, France.

Griffin, D. R. (1971) The importance of atmospheric attenuation for the echolocation of bats (Chiroptera). Anim. Behav. 19: 55-61.

Griffin, D. R., F. A. Webster, and C. R. Michael (1960) The echolocation of flying insects by bats. Anim. Behav. 8: 141-154.

Griffin, D. R., J. H. Friend, and F. A. Webster (1965) Target discrimination by the echolocation of bats. J. Exp. Zool. 158: 155-168.

Grinnell, A. D. (1963) The neurophysiology of audition in bats: Temporal parameters. J. Physiol. (Lond.) 167: 67-96.

Grinnell, A. D., and V. S. Grinnell (1965) Neural correlates of vertical localization by echo-locating bats. J. Physiol. (Lond.) 181: 830-851.

Grinnell, A. D., and H. -U. Schnitzler (1977) Directional sensitivity of echolocation in the horseshoe bats, Rhinolophus ferrumequinum. II. Behavioral directionality of hearing. J. Comp. Physiol. 116: 63-76.

Hartridge, H. (1945) Avoidance of obstacles by bats. Nature 156: 55.

Henson, O. W., Jr. (1965) The activity and function of the middle ear muscles in echolocating bats. J. Physiol. (Lond.) 180: 871-887.

Henson, O. W., Jr. (1967) The perception and analysis of biosonar signals by bats. In Animal Sonar Systems: Biology and Bionics, R. G. Busnel, ed., Vol. II, pp. 949-1003, Laboratoire de Physiologie Acoustique, Jouy-en-Josas, France.

Henson, O. W., Jr. (1970) The ear and audition. In Biology of Bats, W. A. Wimsatt, ed., Vol. II, pp. 181-263, Academic Press, Inc., New York.

Jen, P. H. -S. (1982) Electrophysiological analysis of the echolocation system of bats. Contrib. Sens. Physiol. 6: 111-158.

Johnson, D. H. (1980) The relationship between spike rate and synchrony in responses of auditory-nerve fibers to single tones. $\mathrm{J}$. Acoust. Soc. Am. 68: 1115-1122.

Johnson, R. A., and E. L. Titlebaum (1976) Energy spectrum analysis: A model of echolocation processing. J. Acoust. Soc. Am. 60: 484-491.

Kay, L. (1962) A plausible explanation of the bat's echolocation acuity. Anim. Behav. 10: 34-41.

Kiang, N. Y. -S. (1965) Discharge patterns of single fibers in the cat's auditory nerve. MIT Research Monographs, Boston.

Kick, S. A. (1982) Target detection by the echolocating bat, Eptesicus fuscus. J. Comp. Physiol. 145: 431-435.

Lawrence, B. D., and J. A. Simmons (1982a) Measurements of atmospheric attenuation at ultrasonic frequencies and the significance for echolocation by bats. J. Acoust. Soc. Am. 71: 484-490.

Lawrence, B. D., and J. A. Simmons (1982b) Echolocation in bats: The external ear and perception of the vertical positions of targets. Science 218: 481-483.
Neuweiler, G., V. Bruns, and G. Schuller (1980) Ears adapted for the detection of motion, or how echolocating bats have exploited the capacities of the mammalian auditory system. J. Acoust. Soc. Am. 68: $741-758$

Nordmark, J. (1960) Perception of distance in animal echolocation. Nature 183: 1009

Novick, A. (1977) Acoustic orientation. In Biology of Bats, W. A. Wimsatt, ed., Vol. III, pp. 73-287, Academic Press, Inc., New York.

Pollak, G. D. (1980) Organizational and encoding features of single neurons in the inferior colliculus of bats. In Animal Sonar Systems, R. -G. Busnel and J. F. Fish, eds., pp. 549-587, Plenum Press, New York.

Pollak, G. D., D. Marsh, R. Bodenhamer, and A. Souther (1977) Characteristics of phasic-on neurons in inferior colliculus of unanesthetized bats with observations relating to mechanisms of echo ranging. J. Neurophysiol. 40: 926-942.

Pye, J. D. (1960) A theory of echolocation by bats. J. Laryngol. Otol. 74: 718-729.

Pye, J. D. (1980) Echolocation signals and echoes in air. In Animal Sonar Systems, R. -G. Busnel and J. F. Fish, eds., pp. 309-353, Plenum Press, New York.

Sales, G. D., and J. D. Pye (1974) Ultrasonic Communication by Animals, Chapman and Hall, London.

Schnitzler, II. -U. (1983) The acoustical image of fluttering prey in echolocating bats. In Behavioral Physiology and Neuroethology: Roots and Growing Points, F. Huber and H. Markl, eds., pp. 235-250, Springer-Verlag, New York.

Schnitzler, H. -U., and O. W. Henson, Jr. (1980) Performance of airborne animal sonar systems. I. Microchiroptera. In Animal Sonar Systems, R. -G. Busnel and J. F. Fish, eds., pp. 109-181, Plenum Press, New York.

Shimozawa, T., N. Suga, P. Hendler, and S. Scheutze (1974) Directional sensitivity of echolocation system in bats producing frequency-modulated signals. J. Exp. Biol. 60: 53-69.

Simmons, J. A. (1969) Acoustic radiation patterns for the echolocating bats Chilonycteris ribiginosa and Eptesicus fuscus. J. Acoust. Soc. Am. 46: 1054-1056.

Simmons, J. A. (1973) The resolution of target range by echolocating bats. J. Acoust. Soc. Am. 54: 157-173.

Simmons, J. A. (1979) Perception of echo phase information in bat sonar. Science 204: 1336-1338.

Simmons, J. A. (1980) The processing of sonar echoes by bats. In Animal Sonar Systems, R. -G. Busnel and J. F. Fish, eds., pp. 695714, Plenum Press, New York.

Simmons, J. A., and S. A. Kick (1983) Interception of flying insects by bats. In Behavioral Physiology and Neuroethology: Roots and Growing Points, F. Huber and H. Markl, eds., pp. 267-279, Springer-Verlag, New York.

Simmons, J. A., and R. A. Stein (1980) Acoustic imaging in bat sonar: Echolocation signals and the evolution of echolocation. J. Comp. Physiol. 135: 61-84.

Simmons, J. A., and J. A. Vernon (1971) Echolocation: Discrimination of targets by the bat Eptesicus fuscus. J. Exp. Zool. 176: 315-328.

Simmons, J. A., W. A. Lavender, and B. A. Lavender (1978) Adaptation of echolocation to environmental noise by the bat Eptesicus fuscus. In Proceedings of the Fourth International Bat Research Conference, pp. 97-104, Kenya National Academy of Advancement of Arts and Science, Nairobi, Kenya.

Simmons, J. A., M. B. Fenton, W. R. Ferguson, M. Jutting, and J. Palin (1979a) Apparatus for research on animal ultrasonic signals. Life Sciences Miscellaneous Publications, Royal Ontario Museum, Toronto.

Simmons, J. A., M. B. Fenton, and M. J. O'Farrell (1979b) Echolocation and pursuit of prey by bats. Science $203: 16-21$.

Simmons, J. A., S. A. Kick, B. D. Lawrence, C. Hale, C. Bard, and B. Escudié (1983) Acuity of horizontal angle discrimination by the echolocating bat, Eptesicus fuscus. J. Comp. Physiol. 153: 321-330.

Skolnik, M. I. (1962) Introduction to Radar Systems, McGraw-Hill, New York.

Suga, N. (1964) Recovery cycles and responses to frequency modulated tone pulses in auditory neurons of echolocating bats. J. Physiol. (Lond.) 175: 50-80.

Suga, N. (1970) Echo-ranging neurons in the inferior colliculus of bats. Science 170: 449-452.

Suga, N. (1973) Feature extraction in the auditory system of bats. In Basic Mechanisms in Hearing, A. Møller, ed., pp. 675-744, Academic 
Press, Inc., New York.

Suga, N., and P. H. -S. Jen (1975) Peripheral control of acoustic signals in the auditory system of echolocating bats. J. Exp. Biol. 62: 277311.

Suga, N., and W. E. O'Neill (1979) Neural axis representing target range in the auditory cortex of the mustache bat. Science 206: $351-$ 353.

Suga, N., and P. Schlegel (1972) Neural attenuation of responses to emitted sounds in echolocating bats. Science 177: 82-84.

Webster, F. A. (1967) Some acoustical differences between bats and men. In International Conference on Sensory Devices for the Blind, R. Dufton, ed., pp. 63-87, St. Dunstan's, London.
Webster, F. A., and O. G. Brazier (1965) Experimental studies on target detection, evaluation and interception by echolocating bats. Technical Documentation Report No. AMRL-TR-65-172, Aerospace Medical Division, United States Air Force Systems Command, Tucson.

Webster, F. A., and D. R. Griffin (1962) The role of the flight membrane in insect capture by bats. Anim. Behav. 10:332-340.

Wever, E. G., and J. A. Vernon (1961) The protective mechanisms of the bat's ear. Ann. Otol. Rhinol. Laryngol. 70: 1-13.

Woodward, P. M. (1964) Probability and Information Theory with Applications to Radar, Ed. 2, Pergamon Press, New York. 